\title{
¿De qué están hechos los compuestos?: la interfaz entre constituyentes sintácticos y exponentes léxicos del español
}

\author{
What Kind of Units Constitute a Compound? \\ The Syntax-Lexicon Interface in Spanish
}

BÁrbara Marqueta Gracia

Lingüística General e Hispánica

Universidad de Zaragoza

C/ Pedro Cerbuna, 12. Zaragoza, 50009

marqueta@unizar.es

Orcid ID 0000-0002-2199-1689

Resumen: En el presente artículo se aborda la diversidad morfofonológica de los compuestos del español (manirroto, cuelgacapas, pez estrella) sin recurrir a los conceptos de raíz, tema o palabra, cuyo alcance siempre es limitado para dar cuenta de todas las construcciones que se consideran como compuestas. En su lugar, daremos cuenta de esta diversidad morfofonológica, así como de la distribución de las unidades (en posición interna o externa del compuesto) diferenciando, en primer lugar, dos tipos de contextos sintácticos de inserción del léxico (inserción en proyecciones funcionales activas de concordancia o en contextos sin ellas) y, en segundo lugar, dos tipos de unidades léxicas (libres o ligadas).

Palabras clave: Palabra compuesta. Unidad léxica. Acento. Raíz. Concordancia.
RECIBIDO: 18 DE NOVIEMBRE DE 2016 ACEPTADO: 16 DE FEBRERO DE 2017
Abstract: This paper tackles the morphophonological diversity of Spanish compounds (manirroto, cuelgacapas, pez estrella) without using concepts such as root, stem and word, whose scope is always restricted in order to explain all the constructions typically considered as compounds. Instead, we will deal with such morphophonological variation and distribution of units by differentiating, in first place, two kinds of syntactic contexts of lexical insertion (insertion on contexts with or without active agreement features) and, in second place, two kinds of lexical entries (free and bound entries).

Keywords: Compound Word. Lexical Unit. Stress. Root. Agreement. 


\section{INTRODUCCIÓN}

I asta la fecha, resulta problemático todo intento de caracterizar uniformemente los elementos que integran un compuesto en torno a conceptos como el de raiz (Carstairs-McCarthy 142), tema (Bauer 65) o palabra (Marchand 11).

Cualquier noción de raíz resulta insuficiente para explicar los constituyentes en (1). No cubre la presencia de morfemas derivativos en las unidades subrayadas en (1a) o de marcas de conjugación en las de (1b) -exigidas para la buena formación del compuesto, como demuestra la mala formación de los ejemplos en (1c)-.

1. a. nadador estrella, empresario milagro

b. lavaplatos, vendeppisos

c. *lavplatos, *vendopisos

El concepto de tema, que suele hacer referencia tanto a un conjunto de raíz y categorizador (2a) como a una forma con elementos flexivos sincrónicamente inactivos, como los de caso (2b), excluye a los constituyentes subrayados en las construcciones de (2c), que aparentemente no contienen ni categorizadores ni elementos flexivos.

2. a. videoaficionado, radioyente

b. canceriforme, manufacturado

c. patilargo, blanquiazul

Finalmente, los constituyentes internos de un compuesto presentan, en su mayoría, diferencias respecto a la noción tradicional de palabra morfosintáctica, en tanto en cuanto estos no se flexionan. Ello diferencia a los constituyentes de (1a), retomados como (3a), de los de (1b) y (2), retomados en (3b):

3. a. nadadores estrella, empresarios milagro

b. *lavabaplatos, ${ }^{*}$ videosaficionados, *cancerumforme, ${ }^{*}$ patislargo

Los contrastes recogidos en (4) muestran cómo algunas formas (4a), pero no otras (4b), comparten la posibilidad de participar en la sintaxis oracional, comportándose todas ellas como palabras morfosintácticas dentro de la misma.

4. a. ella lava, dos vídeos, niñas que son verdaderos prodigios

b. "manus, ${ }^{*}$ patis 
Uno de los objetivos de este trabajo es reivindicar la importancia que ser una unidad del léxico del tipo de (4a) adquiere en la formación de compuestos ${ }^{1}$ en aquellas lenguas, como el español, cuyos esquemas compositivos productivos y operativos en sincronía están integrados por tipos diferentes de formas.

Tradicionalmente, la existencia de constituyentes de compuestos que no tienen ni la apariencia ni la distribución de las palabras de la lengua ha constituido una de las razones para justificar su formación en el componente morfológico y no en el sintáctico. Concretamente, la distinción entre compuestos propios y sintagmáticos (De Bustos 69) se ampara en criterios que atañen exclusivamente a la "forma" de las unidades (presencia o no de morfemas flexivos), pero no, por ejemplo, al tipo de categorías que aparecen o de relaciones gramaticales que se establecen en su interior.

Defenderemos que la selección de unidades con la forma de mani, lava o camiones se resuelve en la interfaz entre estructuras sintácticas y exponentes léxicos. Determinados entornos sintácticos exigirán la inserción de unidades léxicas capaces de establecer concordancia, como camiones en los camiones cisterna. Otros exigirán la aparición de formas vinculadas a una categoría (nominal o verbal), pero no se requerirá que dichas formas establezcan relaciones de concordancia, como es el caso de lava en lavaplatos. Finalmente, otros entornos pueden suponer la inserción de formas con propiedades adicionales, como es el caso de mani en manilargo, por lo que la inserción de palabras morfosintácticas de la lengua como camiones sería insuficiente.

La aproximación escogida tiene repercusiones teóricas importantes. Así, algunas de las oposiciones habituales en el grueso de los estudios sobre composición (compuestos formados por raíces frente a compuestos formados por temas) serán desprovistas de relevancia teórica, pues se considerará que únicamente describen el resultado visible del proceso de formación de un compuesto y que, por tanto, no representan las unidades de las que se ha partido para conformar la unidad.

Se asume que el locus de variación entre lenguas se sitúa en el léxico de cada una de ellas -asunción en absoluto novedosa, célebremente conocida como "Borer-Chomsky Conjecture" (Baker 353)-. Por esta razón, la diversidad de formas

1. Aunque no desarrollaremos esta idea, podría añadirse que los exponentes de (4a) son los únicos que participan en otros procesos de formación de palabras sin experimentar ningún tipo de cambio. Así, estos exponentes permiten crear nuevas palabras sin gramaticalizarse y/o lexicalizarse de manera explícita (el celofán desde papel celofán, el busca desde buscapersonas, el ex desde ex pareja, pero nunca *el peli desde pelirrojo). 
observable en los compuestos de cada lengua concreta dependerá de la disponibilidad de exponentes léxicos. Dichos exponentes contienen o no contienen los rasgos gramaticales (p. e. Número) que hacen legítima su inserción en estructuras sintácticas de complejidad variable (la inserción del léxico se rige por principios que detallaremos a lo largo del trabajo). Desde esta perspectiva se puede pensar en la existencia de lenguas en las que, a diferencia del español, solo participen en la formación de compuestos formas como lava y nunca formas como cuelli.

Atendiendo a estas observaciones, vamos a caracterizar tres tipos de compuestos en español en torno a dos criterios: la capacidad de flexionar de las formas que lo integran (que es considerada una propiedad de la entrada léxica) y si dicho potencial flexivo se encuentra activo o inactivo en la estructura del compuesto (propiedad de la estructura sintáctica que se manifiesta o no a través de relaciones de concordancia). El resultado de la caracterización se ejemplifica en la tabla de (5).

5.

\begin{tabular}{|l|c|c|c|}
\hline \multicolumn{1}{|c|}{ TIPO DE CONSTITUYENTE } & camiones cisterna & lavaplatos & Manilargo \\
\hline Potencial flexivo & Sí & Sí & No \\
\hline P.F. activo en composición & Sí & No & No \\
\hline
\end{tabular}

Lo restante del artículo se organiza de la manera siguiente. En el segundo apartado presentaremos el marco teórico de referencia y concretaremos las asunciones teóricas principales de la propuesta. En este mismo apartado caracterizaremos cada una de las unidades compositivas propuestas, delimitando sus contextos de inserción y anticipando las expectativas del modelo de cara a su materialización fonológica. En los apartados tercero y cuarto analizaremos de manera crítica las aproximaciones prosódicas y morfofonológicas a la delimitación de constituyentes compositivos y mostraremos cómo los conceptos presentados pueden contribuir a solucionar algunas de las limitaciones de dichos modelos.

\section{LA INTERFAZ ENTRE SINTAXIS Y LÉXICO Y EL LUGAR}

DE LA MORFOFONOLOGÍA

\subsection{Asunciones teóricas de la propuesta}

Nuestra propuesta asume la hipótesis de la Inserción tardía del léxico de Halle y Marantz (121). La información morfofonológica que diferencia a camión cisterna de lavaplatos o cuellilargo no aparece sobre las estructuras de dichos com- 
puestos hasta que las entradas léxicas identifican dichas estructuras, mediante un procedimiento conocido como Inserción léxica.

Los modelos que asumen típicamente esta hipótesis son los denominados neoconstruccionistas. Dichos modelos presentan diferentes concepciones de la morfología; nuestra propuesta se circunscribe al llamado modelo nanosintáctico, en el cual no existe un componente morfológico que efectúe cambios en las formas de los exponentes léxicos de manera posterior a su inserción, como en la Morfología Distribuida; en Nanosintaxis, la variación morfológica se deriva directamente de los principios que regulan la inserción de exponentes léxicos, presentados de manera exhaustiva en Fábregas (2016b, 44-68).

La implicación más relevante de una propuesta neoconstruccionista para el presente artículo es que la distinción morfofonológica entre raíces, temas y palabras no depende ya de propiedades inherentes a cada tipo de unidad, sino de las estructuras.

No compartimos la asunción de que el elemento que vincula la información semántica idiosincrásica a la estructura sintáctica es una raíz acategorial, sino un elemento léxico capaz de identificar una categoría. Estos elementos léxicos (casa, y no cas-) constituyen, de este modo, la unidad mínima capaz de establecer correspondencia con el Sistema Conceptual y recibir un significado arbitrario, oponiéndose a las categorías funcionales como de, que no denotan conceptos. Adoptaremos el modelo de entrada léxica propuesto por Starke (2), de manera que cada entrada léxica estará integrada por información fonológica, conceptual y sintáctica de tamaño variable.

Entre los elementos del léxico que pueden establecer correspondencia con un concepto, que denominaremos unidades léxicas, diferenciaremos dos tipos: aquellas que pueden recibir materialización fonológica en la lengua de manera independiente, a las que denominaremos unidades léxicas libres, y aquellas que no pueden recibir materialización fonológica de manera independiente, a las que denominaremos unidades léxicas ligadas.

Asumimos que la información que permite distinguir entre formas libres y ligadas forma parte del conocimiento léxico de los hablantes de la lengua. Dicha información forma parte fundamentalmente, aunque no exclusivamente, como puntualizaremos a continuación, de los rasgos fonológicos presentes en la entrada léxica (los mismos que nos permiten evaluar snappish como una palabra no perteneciente a nuestra lengua). Hablaremos de unidades léxicas ligadas en oposición a afijos o clíticos, que serían unidades funcionales ligadas. 
Un aspecto determinante para la independencia fonológica, como es la presencia o no de un acento principal, no se considerará determinado por la información fonológica del léxico, sino por su contexto sintáctico de inserción, dado que asumimos que la asignación de acento se produce sobre la estructura sintáctica de manera posterior a la inserción léxica. De esta manera, en nuestra propuesta habrá formas como navarro que serían unidades libres en contextos como el pueblo navarro, pero unidades ligadas en contextos como pueblo navarro-aragonés.

Hemos identificado las siguientes generalizaciones o tendencias en la materialización fonológica de compuestos, según el tipo de unidades léxicas (libres o ligadas) que aparecen en su interior:

a) Los compuestos en los que se han materializado fonológicamente dos unidades léxicas ligadas, como herbivoro, presentan siempre evidencias de interdependencia fonológica, como son la ausencia de diptongo en herbi y la asignación de un único acento en la construcción.

b) Los compuestos en los que se han materializado fonológicamente dos unidades léxicas libres, como azul cielo, no presentan ningún tipo de interdependencia fonológica, como evidencia la aparición de diptongo en cielo y la asignación de dos acentos principales en la construcción.

Los compuestos formados por una combinación de unidades léxicas ligadas y libres, como pernilargo o cuelgacapas, presentan propiedades morfofonológicas y prosódicas híbridas respecto a los anteriores, aunque cabe distinguir dos subtipos, dependiendo del tipo de exponente léxico que se introduce en la posición interna del compuesto.

a) Cuando la unidad léxica ligada lo es de tipo inherente (la dependencia fonológica está condicionada por sus rasgos fonológicos), como perni en pernilargo, el compuesto comparte propiedades en su materialización fonológica con el tipo de herbivoro, como es la presencia de monoptongo en perni. Sin embargo, el patrón de asignación de acento es claramente diferente entre ambos compuestos, pues herbi recibe el acento en herbivoro pero perni no lo hace en pernilargo.

b) Cuando la unidad léxica ligada lo es de manera reciclada (la dependencia fonológica está condicionada por el contexto de inserción), como cuelga en cuelgacapas, el compuesto comparte propiedades en su 
materialización fonológica con el tipo de azul cielo, como es la presencia de diptongo en cuelga, aunque nuevamente el patrón de asignación de acento es claramente diferente (tanto azul como cielo reciben acento principal en azul cielo pero la forma diptongada cuelga no lo hace en cuelgacapas).

Resumimos estas observaciones en el cuadro de (6). Dicho cuadro refleja cómo unidades similares, pero en diferentes contextos de inserción, presentan propiedades diferentes en lo que respecta a su materialización fonológica.

6.

\begin{tabular}{|c|c|c|c|c|}
\hline $\begin{array}{c}\text { TIPO DE } \\
\text { cOMPUESTO }\end{array}$ & $\begin{array}{c}\text { u. libres } \\
\text { (cuento estrella) }\end{array}$ & $\begin{array}{c}\text { u. reciclada + libre } \\
\text { (cuentoterapia) }\end{array}$ & $\begin{array}{c}\text { u. ligada + libre } \\
\text { (corniabierto) }\end{array}$ & $\begin{array}{c}\text { u. ligadas } \\
\text { (cornifero) }\end{array}$ \\
\hline $\begin{array}{c}\text { Dos } \\
\text { acentos } \\
\text { principales }\end{array}$ & Sí & Infrecuente & No & No \\
\hline Diptongos & Sí & Sí & Infrecuente & No \\
\hline $\begin{array}{c}\text { Acento } \\
\text { principal } \\
\text { en } \\
\text { u. interna }\end{array}$ & Sí & No & No & Sí \\
\hline
\end{tabular}

En nuestra propuesta se combinan aspectos de los modelos morfológicos "basados en el lexema" en lo que respecta a las unidades de almacenamiento léxico con aspectos de los modelos "basados en el morfema" en lo que respecta a la complejidad de las unidades morfológicas. Aceptamos la asunción típica de los modelos neoconstruccionistas de que la palabra compuesta tiene complejidad sintáctica -siendo dicha complejidad interpretable en la estructura de manera posterior a la inserción léxica-.

El hecho de que unidades de mayor tamaño que una raíz deban almacenarse en el léxico del español se justifica con contrastes como los de (7). Elementos con una misma raíz (cesto/cesta) se relacionan con conceptos distintos en su implementación como sustantivos, pese a que elementos funcionales como los clasificadores o las vocales temáticas no poseen, en teoría, propiedades que pudieran alterar el contenido semántico de las raíces, a diferencia de un afijo como-dor, por ejemplo.

7. a. cesto-cesta

b. regentar-regir-reinar

c. dependiente-dependienta 
Asumimos la hipótesis del almacenamiento léxico de temas (Bermúdez 22). ${ }^{2}$ Según el autor, formas como cesto y cesta constituyen distintos objetos de almacenamiento léxico y se predice, por tanto, el hecho de que se relacionen con conceptos diferentes. Esta hipótesis sería suficiente para explicar el almacenamiento de compuestos enteros (cuellilargo, guardarropa, etc.) pero no nos permite explicar por qué un compuesto como cuellilargo se almacena con la forma cuelli y no con la forma cuello, dado que cuelli y cuello son alomorfos que remiten a un mismo concepto.

La hipótesis del almacenamiento de compuestos enteros, a nuestro entender, explica sin mayor inconveniente la presencia en compuestos de alomorfos absolutamente improductivos como los de (8). En la formación sincrónica de compuestos no se recurre a este tipo de alomorfos.

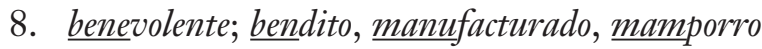

Pero el recurso a los principios nanosintácticos de inserción léxica está plenamente justificado en aquellos casos como los ejemplificados en (9). En la formación, totalmente productiva en sincronía, de compuestos como lavaplatos o derivados como amable nunca aparece en posición interna un elemento léxico sin vocal temática (9).

El principio nanosintáctico de lexicalización exhaustiva nos permite explicar la agramaticalidad de los ejemplos sin vocal temática, dado que dicho principio predice que todos los rasgos sintácticos de una estructura tienen que ser identificados por el léxico. Exponentes como mato dejarían los rasgos verbales sin identificar en la estructura de las formaciones porque carecen de vocal temática.

\section{9. a. cazafantasmas, vendepisos; *matomoros amable, bebible; *beboble}

A lo largo del trabajo se añadirán nuevos casos y se detallarán contextos similares a los de (9). Todos ellos muestran que los contextos sintácticos de inserción del léxico nos permiten predecir en gran medida la aparición de las formas de los compuestos.

Por motivos de espacio, no abordaremos cuestiones relativas a la organización interna del componente léxico. Ello nos obliga a dejar abiertas algunas

2. El autor se apoya en evidencia empírica, pues los efectos de frecuencia ofrecen resultados significativamente distintos para las alternancias (listadas) de género frente a las (no listadas) de número, p. ej. Domínguez/Cuetos/Segui (488-90). 
cuestiones, por ejemplo: ¿cómo se accede a cada una de las formas? Independientemente de cómo respondamos estas cuestiones, la actividad de comprensión y producción léxica desencadenada por los neologismos de (10) debe ser sensible a los condicionantes sintácticos (categoriales) de la distribución de las formas, puesto que estos condicionantes actúan como un filtro de la potencial proliferación de alomorfos (rompi, rompa, etc.) dentro de un mismo compuesto.

10. b. rompepactos, insultadiputados

\subsection{Los contextos de inserción léxica de constituyentes compositivos}

Hasta la fecha, es habitual que los monográficos y tesis doctorales que versan sobre la composición en español organicen en apartados o capítulos diferentes la morfofonología y la sintaxis de los compuestos. Se distinguen tipos compositivos en torno a sus propiedades categoriales (compuestos del tipo de $\mathrm{N}+\mathrm{N}$, $\mathrm{V}+\mathrm{N}$, etc.) o según sus relaciones gramaticales (compuestos coordinantes, subordinantes o atributivos). Sin embargo, no es habitual encontrar propuestas donde se analicen de manera sistemática el tipo de, por ejemplo, sustantivos, que aparecen en el interior de compuestos. El resultado sería una tipología como la de (11): en la columna de la izquierda se recogen sustantivos con diferentes propiedades morfofonológicas y en la columna de la derecha sus propiedades gramaticales.

11. a. Los $\underline{\text { camiones }} /{ }^{*}$ camion cisterna $\quad \mathrm{N}$, género, número (concordancia) m.pl m.pl *m.s f.s

b. el buscapersonas; portavoz m.s f.pl f.s

c. la videoaficionada; carilinda

N, número (semánticamente interpretable $)^{3}$

$\mathrm{N}$

f.s m.s

Para cada tipo de unidad léxica que participa en la formación de un compuesto:

- Presentaremos las formas representativas de cada unidad.

- Detallaremos el contexto sintáctico en el que se insertan.

- Anticiparemos algunos aspectos relativos a su materialización fonológica.

3. El plural de camiones cisterna y lavaplatos es semánticamente interpretable (hay más de un camión y se lava más de un plato). En el caso de videoaficionado, no: un videoaficionado puede ser aficionado a un único tipo de vídeo, a los vídeos en general, etc. 


\section{a. Unidad léxica libre}

Esta clase está representada por todos aquellos miembros de una categoría léxica flexiva que aparecen en un compuesto pudiendo establecer una relación de concordancia manifiesta. Se incluyen por tanto sustantivos (12a), verbos (12b) o adjetivos (12c) como los subrayados.

12. a. los camiones cisterna, la niña prodigio, otra videollamada

b. Mientras ellos manuscribian el boceto, ella fotocopió el original

c. Las jóvenes teleadictas eran pelirrojas

En este trabajo sintagmas y compuestos se consideran estructuras sintácticas. El criterio que empleamos para diferenciar ambas estructuras en casos problemáticos como los dos primeros ejemplos de (12a) es el nivel de la estructura en el que se producen las relaciones de concordancia. La relación de concordancia establecida en un compuesto es siempre posterior al ensamble (unión) de las dos unidades léxicas (13a) y no se produce durante el mismo (13b). Si se produce una relación de concordancia durante el ensamble de dos unidades léxicas libres, lo que se proyecta es un sintagma y no un compuesto (13b).

13. a.

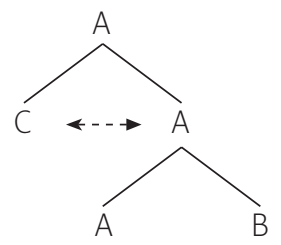

$\mathrm{C}=$ los $\quad \mathrm{A}=$ camiones $\quad \mathrm{B}=$ cisterna b.

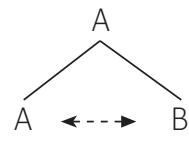

$\mathrm{A}=$ osos $\quad \mathrm{B}=$ polares

La figura de (13a) representa un compuesto: camiones se ensambla con cisterna y, solo posteriormente, camiones concuerda con los. La figura de (13b) representa un sintagma: osos se ensambla con polares y entre estos dos elementos ya se establece concordancia.

La estructura de (13a) es únicamente posible en los compuestos de núcleo sustantivo. Creemos que la explicación de este hecho radica en que la categoría funcional determinante forma parte de la proyección funcional del sustantivo (Abney 277). De esta manera, el ensamble entre camión y cisterna da lugar a otra proyección de sustantivo, cuyo núcleo es camión, que puede proyectar hasta determinante, como en el camión (14a). Sin embargo, ni el verbo co- 
pió ni el adjetivo adictas, que también pertenecen al bloque de ejemplos de (12), establecen una relación de concordancia con una categoría funcional que forme parte de su proyección (tiempo, grado): lo hacen con un sintagma nominal, proyección de una categoría léxica distinta, como se ejemplifica en (14b). En este tipo de circunstancia, el elemento con el que concuerda el núcleo no forma parte del compuesto (14c).

14. a.

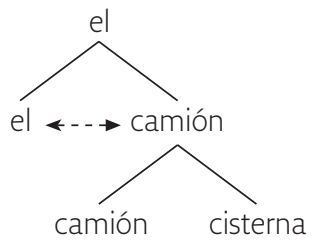

el camión cisterna b.

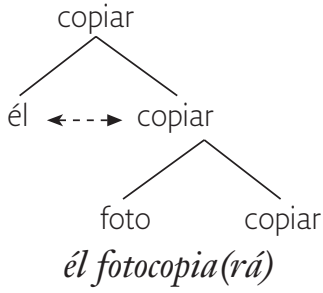

c. *

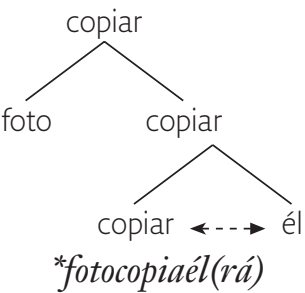

La no aparición de sujetos en el interior de los compuestos verbales constituye una observación ya antigua en los trabajos sobre la composición (Rainer/Varela 128), por lo que queda excluida la posibilidad de una concordancia entre sujeto y verbo. En lo que respecta a las formaciones con adjetivos como aguas mayores, la relación de concordancia se produce entre los dos constituyentes léxicos (agua y mayor) durante su ensamble. Hemos considerado que este, entre otros motivos, justifica el tratamiento de estas construcciones como sintagmas (con significado idiomático) y no como palabras compuestas. $^{4}$

Convenientemente, hemos evitado subrayar cisterna y prodigio en camión cisterna y niño prodigio en (12a) y habríamos evitado subrayar platos en lavaplatos. Pese a no establecerse ninguna relación de concordancia visible ni dentro del compuesto ni fuera del mismo, presentan propiedades (acento principal) que hacen difícilmente justificable su exclusión del conjunto de las unidades léxicas libres.

4. Los compuestos como pez globo tienen significado composicional en un sentido del cual carecen formaciones como aguas mayores; concretamente, la interpretación del núcleo es siempre composicional, así, un camión cisterna siempre es un camión pero aguas mayores no es un tipo de agua ni cabello de ángel un tipo de cabello. La misma conclusión se alcanza en Mendívil (105), analizando camión cisterna como estructura composicional y reanalizada. El autor mantiene una estricta separación morfofonológica entre compuestos léxicos y sintagmáticos, pero creemos que sus observaciones podrían implementarse satisfactoriamente sobre los compuestos léxicos también. 
La solución que proponemos es que, efectivamente, estas formas son unidades léxicas libres que podrían establecer relaciones de concordancia. Ello se evidencia en la posibilidad de reanalizar construcciones como reyes magos como sintagmas integrados por un sustantivo y un adjetivo y la variación en construcciones como pisos piloto(s).

La concordancia con un determinante que experimentan los núcleos sustantivos como camión no se va a manifestar en los sustantivos no-núcleos porque todos los no-núcleos de los compuestos del español carecen sistemáticamente de la proyección funcional de determinante. La ausencia de determinante no es una propiedad exclusiva de aquellas construcciones, como los compuestos, que podrían llamarse "morfológicas", dado que sustantivos no-núcleos en contextos claramente sintagmáticos también carecen de ellos (15):

15. a. Le tiró (*los) besos...

*fregalossuelos

b. una pintura con efecto (*la) arena

*camionlacisterna

En resumen, las unidades léxicas libres se insertan siempre que en un compuesto de núcleo verbal o adjetival se requiere del establecimiento de una relación de concordancia de persona o número con un sujeto, en cuyo caso las unidades léxicas libres se materializarán en la posición externa del compuesto; en un compuesto aparecen sustantivos que contienen proyecciones de género y número, condición necesaria, pero no suficiente, para que se establezca concordancia con una proyección de determinante.

Amén de las propiedades morfosintácticas descritas, que tienen un claro correlato morfofonológico, las unidades léxicas libres se caracterizan por ser siempre portadoras de un acento principal en su materialización fonológica en español.

\section{b. Unidad léxica ligada}

Esta categoría está representada por diferentes tipos de unidades léxicas, entre ellas, categorías no flexivas con significado conceptual (16a), antiguas categorías flexivas cuyos rasgos se encuentran sincrónicamente inactivos (16b) o categorías léxicas que pierden sus rasgos flexivos al ensamblarse con una categoría funcional -con un valor similar a preposiciones o conjunciones- (16c). Estas unidades tienen en común el estar relacionadas, bien por su etimología, 
bien por su distribución y significado, con categorías relacionales como preposición, conjunción o caso.

Así, anti en (16a) introduce como su término aquello de lo que no se es partidario, como la preposición contra. Entre es una preposición de la lengua. Los sustantivos subrayados en (16b) presentan una vocal de cierre vinculada etimológicamente a lenguas que flexionaban caso en sus sustantivos. Finalmente, los sustantivos de (16c) están significativamente vinculados al acusativo de relación, según Lapesa (87).

16. a. antisistema, entreacto

b. arboricidio, manuscrito

c. cuelli (cuello+i), blanqui (blanco+i)

En el sistema que proponemos, sintagmas como blanco y azul nunca experimentan una situación de competencia con compuestos como blanquiazul, contra Ackema/Neeleman (48), en cuyo modelo sintagma y compuesto sí competirían en la materialización de esta estructura. La inserción de las unidades léxicas libres como blanco se produce en una proyección con rasgos de concordancia que han de identificar ambos adjetivos (no solo azul, el más externo). Así, en el sintagma, únicamente la unidad léxica libre blanco, pero no blanqui, es insertable (pantalones blancos y de buen gusto/*blanqui y de buen gusto).

Las unidades léxicas ligadas se caracterizan por no poder materializarse de manera independiente en la lengua (17a) y por no recibir acento principal cuando se adjuntan a palabras léxicas libres (17b). ${ }^{5} \mathrm{Al}$ carecer de independencia prosódica en su materialización, es habitual que aparezcan monoptongos (17c), pues existe un vínculo entre acentuación y diptongación, como mantienen los modelos que veremos en el próximo apartado. Las bases ligadas pierden su vocal de tema cuando se adjuntan a un afijo vocálico, fenómeno que es fácilmente observable en derivación (17d), pero no así en composición (dado que lo que se adjunta a quita en quitaesmaltes no es un afijo).

17. a. *los antis, *los entres, *las manus, *las blanquis

b. antisistéma, drogodependiénte, cuellilárgo, blanquiazúl

c. dentimellado, dentera, dentífrico

d. arenoso/arenas; matón/matador

5. Tenemos en cuenta la existencia de unidades acentuadas como antídoto, pero debe notarse que aquí anti- se adjunta a otra unidad léxica ligada, lo que cambia las condiciones de asignación de acento. 


\section{c. Unidad léxica libre reciclada como ligada}

Esta categoría está representada por todas aquellas unidades léxicas libres de la lengua que aparecen en la posición interna de un compuesto en español, a excepción del núcleo de compuestos como peces globo.

Las unidades recicladas se diferencian de las unidades léxicas libres, al no poder proyectar sus propiedades flexivas ni establecer relaciones de concordancia. ${ }^{6}$ Así, la forma conjugada que aparece en el compuesto verbonominal nunca habilita un sujeto (18a), mientras que las unidades libres de la misma categoría sí lo hacen (18b). La forma nominal reciclada que aparece en el compuesto de (18c) nunca flexiona en número, a diferencia de las unidades libres de (18d), que sí pueden hacerlo.

18. a. vendesueños (*vende-él sueños)

b. Ellos compran todo y luego lo malvenden

c. videojuego ( ${ }^{*}$ videosjuegos)

d. videollamadas, ${ }^{*}$ camiones cisternas pero perros lobos

La inserción léxica de unidades recicladas se produce en contextos donde no pueden aparecer bases inherentemente ligadas, como es el caso del patrón verbonominal de lavaplatos, pero también en contextos donde sí coaparecen (aerosolterapia y vitaminoterapia).

Las unidades recicladas pueden concebirse como palabras fonológicas que pierden su acento en la derivación; esta es la aproximación que favorecen los trabajos que veremos en el próximo apartado. También pueden concebirse como unidades almacenadas en el léxico, resolviéndose sus características prosódicas de manera posterior. Nos hemos decantado por esta última posibilidad porque la reestructuración prosódica no afecta en particular a las palabras fonológicas, sino a cualquier elemento lingüístico que es reciclado como ligado. La reestructuración ocurre también en sintagmas fonológicos, como evidencian guerracivilista, treintañero o altoaragonés.

La única particularidad fonológica de las unidades recicladas es la ausencia de acento principal, que nos permite distinguirlas de las unidades libres (19a). Unidades libres y recicladas presentan, por lo demás, propiedades comunes, como la presencia de diptongos (19b).

6. Un revisor anónimo comenta que la condición de no-núcleo de algunas formas en (18) puede explicar la ausencia de propiedades flexivas. 
19. a. Láva plátos. Làvaplátos; Al vídeo juégo. Al vìdeojuégo

b. cuelgacapas como huelga sorpresa o Lavapiés

Recapitulemos brevemente lo visto en este apartado. La exploración de los contextos de inserción de exponentes léxicos nos ha permitido comprobar que la morfofonología de cada categoría (sustantivo, adjetivo, verbo) varía en función de las exigencias de las estructuras. Los entornos con concordancia exigen la aparición de unidades léxicas libres (palabras morfosintácticas en el sentido tradicional), mientras que los entornos con categorías relacionales exigen la inserción en español de una unidad léxica ligada de manera inherente (diferente a las raíces o los temas tradicionales). Hemos justificado las particularidades de la única construcción con dos unidades léxicas libres, representada por camiones cisterna. En los compuestos restantes (lavaplatos) las unidades insertadas desde el léxico no identifican proyecciones de concordancia en posición interna. De hacerlo, la proyección sería sintagmática (Lavan platos).

En (19a) observábamos que la presencia de acento principal permite distinguir sistemáticamente unidades libres y recicladas. Desde un modelo de Inserción Tardía resultaría contradictorio que un criterio fonológico (la presencia de acento) condicionara una distinción estructuralmente pertinente (presencia o no de concordancia). Es por ello que parece necesario abordar la cuestión desde la perspectiva inversa, de manera que sean los contextos de inserción, y no los exponentes, los que determinen la posibilidad de asignación de acento.

En la introducción de este trabajo afirmábamos que la condición de ligada de una unidad léxica podía vincularse tanto a propiedades fonológicas de la forma almacenada como al contexto de inserción léxica de dicha forma. Consideramos que la naturaleza ligada con la que escribe se integra de manera inmediata en un neologismo como lápiz escribecartas representa este segundo tipo de dependencia. En español, la posibilidad de que una unidad en posición interna reciba acento principal correlaciona con la posibilidad de dicha forma de establecer relaciones de concordancia (camiones cisterna).

\section{LA ESTRUCTURA FONOLÓGICA DE LAS PALABRAS COMPUESTAS}

En este apartado presentaremos algunas de las propuestas que abordan la distinción de los constituyentes compositivos del español mediante criterios fonológicos y prosódicos. Comentaremos las ventajas e inconvenientes de cada una de ellas, señalando la manera en que sus predicciones empíricas son compartidas o no en el sistema que hemos propuesto. 
En Elordieta (7-16) encontramos una nómina exhaustiva de los criterios utilizados para delimitar palabras fonológicas desde un punto de vista prosódico. La idea relevante de cara a la delimitación de constituyentes compositivos es que podemos encontrar diferentes tipos de unidades prosódicas dentro de un compuesto, algunas de las cuales no constituyen palabras prosódicas por sí mismas, sino que lo hacen conjuntamente con otros elementos.

Así Elordieta diferencia compuestos integrados por dos bases fonológicas, resultando en una palabra prosódica (20a); compuestos integrados por base fonológica y palabra prosódica, dando lugar a otra palabra prosódica (20b) y compuestos integrados por dos palabras prosódicas, constituyendo un sintagma fonológico (20c).

20. a. bió+grafo, mani+curo

b. lava+platos, drogo+dependiente, mani+rroto

c. camiones+cisterna, coches+escoba

En (20) quedan reflejados los tipos de materialización fonológica que hemos vinculado a unidades léxicas ligadas (20a), libres (20c) y a una combinación de ambas (20b), aunque se pasa por alto la afinidad formal entre exponentes como lava con las unidades libres de (20c) y la de exponentes como mani con las unidades ligadas de (20a).

Esta propuesta pone en evidencia un aspecto que consideramos de fundamental importancia, como es el hecho de que ninguno de los compuestos de (20) presente diferencias de integración fonológica de acuerdo a criterios como los que ofrece el propio Elordieta (12 y 38-45). Observamos, por ejemplo, que ninguno de los constituyentes internos de un compuesto interdentaliza el fonema $/ \mathrm{k} /$ ante glide (21).

21. anquiseco-*anciseco; blaquiazul-*blancizul

ciudad isla * ciudacisla

(frente a clasicidad o misticismo)

Si ciudad isla no se diferencia de blanquiazul en función de estos fenómenos, el único criterio pertinente para distinguir entre compuestos desde la perspectiva prosódica es, nuevamente, el acentual. Hechos como los de (21), por tanto, apoyan indirectamente nuestra hipótesis de que la distinción entre tipos de compuestos no se puede amparar en propiedades prosódicas inherentes a las unidades de input.

En resumen, la integración fonológica entre constituyentes con repercusiones morfofonológicas visibles es excepcional en todo tipo de compuestos, 
claramente el producto de procesos de lexicalización (en su dimensión histórica) que operan sobre formaciones concretas como telaraña (tela-arácnea) o vinagre (vino-acre). Este tipo de integración se produce, también muy esporádicamente, en los compuestos léxicos: paraguas, manvacío.

\subsection{La materialización fonológica independiente de constituyentes compositivos}

La ausencia de integración fonológica entre las unidades que conforman un compuesto ha conducido a algunos a autores en el seno de la Morfología Distribuida a proponer que dichas unidades se materializan por separado. La primera referencia que encontramos a esta posibilidad es Harley (224), aplicada a compuestos que contienen un sintagma como bikini-girls-in-trouble genre.

La materialización fonológica de las estructuras sintácticas se produce de manera cíclica en estos modelos. Predicen que los elementos que son materializados en un mismo ciclo presentan unas propiedades fonológicas que evidencian su materialización conjunta, como puede ser el hecho de que un mismo sufijo se materialice como -ción en manifestación y como -ión en visión.

Los modelos de materialización cíclica presentan algunos problemas de implementación en las palabras compuestas. Predicen correctamente que los constituyentes de palabras como cuentabílos no presentan las propiedades que cabría esperar si su materialización se produjera en un mismo ciclo, en primer lugar, la forma cuenta aparece diptongada. Lo esperable en un ciclo conjunto de materialización de cuentabílos es que no lo estuviera, al no recaer el acento principal sobre la $o$ subyacente al diptongo ue (igual que en contarémos). En segundo lugar, no se produce una integración fonológica que resulte en *cuentilos (que sí se produce en cuentita). Sin embargo, la desventaja de separar cíclicamente la materialización de cuenta de la de bilos es que se crea una asimetría con el dominio de asignación conceptual, puesto que parece evidente que la suma de los significados de cuenta e bilos por separado no nos permite deducir que un cuentabílos es un tipo de microscopio. Una manera de solventar este tipo de inconveniente sin renunciar por ello a un modelo cíclico consiste en retrasar la materialización más allá del primer tema (ver Bermúdez 63-69).

Un análisis cíclico aplicado a los compuestos verbonominales del español lo encontramos en Shwayder (5). La figura (22) recoge el estatuto de palabra fonológica de los dos constituyentes (reanalizados como una única raíz) de manera previa al proceso de nominalización del compuesto. La motivación de separar estos compuestos en dos ciclos de materialización reside para el 
autor en el hecho de que están integrados por dos palabras fonológicas. El estatuto de palabra fonológica del primer miembro se justifica por la presencia de diptongos (23a) y por la falta de integración fonológica (23b).

22.

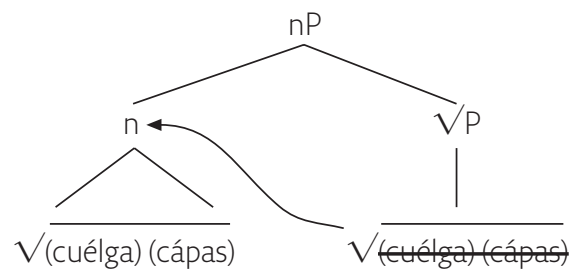

(Shwayder 5)

23. a. cuelgacapas (*colgacapas), muerdealmohadas (* mordealmobadas)

b. quitaesmaltes (*quitasmaltes), guardaespaldas ( ${ }^{*}$ guardaspaldas)

Los diptongos de (23a) se explican como el resultado de un ciclo de diptongación sobre el tema verbal. ${ }^{7}$ Debemos suponer que solo este último criterio es necesario para justificar la condición de palabra fonológica de las formas, pues cabe recordar que en otros compuestos no suelen diptongar formas -corniapretado, dentimellado- que no se diferencian de las de (23b) en lo que respecta a la ausencia de integración fonológica (culi-alto con hiato).

Desde nuestra propuesta, las unidades libres recicladas como cuelga se encuentran diptongadas de manera previa a su inserción léxica, lo que nos permite desvincular la presencia del diptongo de la asignación de un ciclo de acentuación en el compuesto. Es cierto que la pérdida de acento principal en cuelga se puede explicar como el resultado de la reestructuración prosódica del compuesto, pero resulta en cierta manera paradójico que la estructura compositiva sea, en la propuesta del autor, la encargada de provocar simultáneamente el ciclo de acentuación del compuesto y su posterior desacentuación.

En Fábregas (2016a, 10-15) también se relaciona la presencia de diptongos con un ciclo de materialización. Dicho ciclo es experimentado por las formas subrayadas en (24a), no así por las de (24b).

24. a. bierba-adicto, concierto-adicto

b. berbicida, concertófilo

(Fábregas 2016a, 7-8)

7. Coincidiendo con la larga tradición de análisis de estas formas. 
Según el autor, formas como bierba contienen una vocal temática, constituyendo por ello un "especificador complejo". Los especificadores complejos son transferidos y materializados fonológicamente (Uriagereka 253). La transferencia no se produciría con una forma como herb-, que es únicamente una raíz y, por tanto, no constituye un dominio de materialización. La $i$ en herbies únicamente una vocal de enlace que crea un punto de asimetría para que se pueda producir la fonologización del compuesto.

Hay varias razones para no aceptar este análisis. En primer lugar, la reducción que experimenta droga en drogadicto (que no *drogaadicto) evidencia un mínimo de integración, lo que obligaría a estipular la aplicación de una regla de reajuste de manera posterior a la materialización de adicto, lo cual debería ser imposible si droga ya ha sido transferido y materializado. En segundo lugar, la presencia de monoptongos en formas como herbi- tiene, como es lógico, una explicación etimológica alternativa. El autor señala la mala formación de *bierbicida, pero esta no puede deberse a la presencia del diptongo: a la hora de dar nombre al asesinato de una suegra, tanto socricidio como suegricidio suenan perfectamente aceptables, con aparente preferencia por la segunda.

El principal motivo para no aceptar la presencia de un ciclo de materialización en compuestos como los de (24a) es que el fenómeno no sería todo lo uniforme y sistemático que podríamos esperar de un ciclo. En la posición interna de los compuestos de (25) aparecen, efectivamente, palabras de la lengua (25a), pero también temas cultos (25b) y léxico patrimonial asimilado al esquema latinizante (25c). La pregunta que surge inmediatamente es si tiene sentido postular un ciclo de materialización que pueda aplicarse con algunas de las palabras recogidas en (25), como las de (25a), pero no con todas las demás. ${ }^{8}$

\section{5. a. aromaterapia, aerosolterapia \\ b. bidroterapia, helioterapia \\ c. dietoterapia (dieta), risoterapia (risa)}

En una aproximación como la nuestra, la selección de alomorfos en los casos de (25) no está restringida por los contextos de inserción, pues dependerá de

8. Dicha variación no es habitual en los compuestos con -adicto, cuya productividad actual se ve estimulada por la introducción de calcos del inglés (aunque sí está documentada músico-adicto para referir a la adicción a la música, que no a los profesionales de la misma). 
las preferencias de cada hablante concreto, de la disponibilidad de unidades ligadas de inspiración clásica en su léxico y de factores extralingüísticos como el tipo de voz creada: si es un tecnicismo, por ejemplo, es más probable que se seleccionen alomorfos como los de (25b-c).

Recapitulemos brevemente. En este apartado se han señalado los inconvenientes de la implementación de ciclos de materialización en el interior de palabras compuestas, que son fundamentalmente dos: el desmembramiento de un objeto lingüístico que debe recibir una interpretación conceptual de manera conjunta y la falta de sistematicidad de las propiedades fonológicas de las que la existencia de un ciclo en el interior del compuesto pretende dar cuenta. Retomaremos estas cuestiones en el cuarto apartado.

\subsection{La caracterización fonológica en torno a la distinción compuesto léxico y compuesto sintagmático}

En inglés, los compuestos se acentúan típicamente en el constituyente situado a la izquierda, por lo que la posición del acento constituye un criterio habitual en la identificación de compuestos frente a sintagmas. La "regla de acentuación", sin embargo, no se aplica a todas las construcciones que se pueden considerar compuestos. Remitimos a Lieber/Stekauer (1-25) para una identificación y explicación detallada de las aparentes excepciones, entre las que se incluyen las de (26).

\section{6. hand-máde 'manufacturado'} woman dóctor 'mujer doctor' summer-night 'noche de verano'

\section{(Lieber/Stekauer 8-12)}

Hualde (59-89) establece un paralelismo entre la distribución de acentos en los compuestos del español y el inglés. Las construcciones acentuadas en el constituyente izquierdo en inglés tendrían su correlato estructural en español con aquellas donde se acentúa el constituyente derecho, a saber, los compuestos léxicos de (27a), mientras que las construcciones acentuadas en inglés en el constituyente derecho corresponderían a las construcciones con doble acento o los comúnmente llamados compuestos sintagmáticos (27b).

27. a. matasuégras, drogodependiénte, blanquiazúl, sobremésa

b. péz burbúja; ensaladílla rúsa 
El principal problema lo supone la supuesta existencia de casos ambiguos entre las dos pautas de acentuación (28) y su consideración como construcciones a su vez ambiguas en su condición de sintagmas y compuestos.

\section{8. fronteras paraguayo-bolivarianas operaciones político-militares}

\section{(Hualde 75)}

Los casos de (28) son verdaderamente problemáticos para la distinción entre palabras y sintagmas como objetos morfofonológicos con propiedades distintivas. Desde una aproximación como la nuestra, en la que sintagmas y compuestos se distinguen en torno a criterios estructurales, los casos de (28) constituyen únicamente compuestos.

La aparición (no ambigua o, al menos, no controvertida) de dos o más acentos principales, como en los ejemplos de (29), viene acompañada de propiedades estructurales que nos permiten identificar sintagmas. En el caso de (29a) encontramos un sintagma con un modificador integrado por adjetivos en yuxtaposición; en el caso de (29b), una aposición cuyo segundo miembro contiene dos sustantivos yuxtapuestos.

29. a. diccionario enciclopédico-crítico-etimológico *fronteras paraguayo-bolivariana-argentina

b. relaciones bijo-madre maltratada *operaciones político-[militares extremistas]

El sintagma de (29a) no es necesariamente bimembre y facilita una interpretación semántica aditiva 'diccionario enciclopédico y también crítico y también etimológico’. La coordinación de adjetivos en compuestos (28) es bimembre y de interpretación semántica preferiblemente intersectiva 'fronteras entre Paraguay y Bolivia'. En (29b) notamos como uno de los modificadores (madre) se puede modificar, lo que nunca ocurre en compuestos con dos adjetivos coordinados (28).

La expectativa es que si político fuera una unidad léxica libre -con potencial de concordancia- establecería una relación de concordancia con operaciones, proyectándose el sintagma operaciones políticas. Siendo un sintagma, este se podría expandir indefinidamente (las operaciones políticas rusas innecesarias) (30a). Pero esto nunca sucede, porque el político de político-militares es una unidad léxica ligada que nunca establece una relación de concordancia con operaciones (30b). 
30. a.
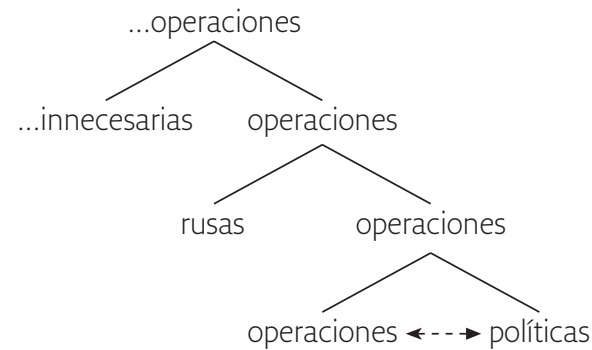

b.

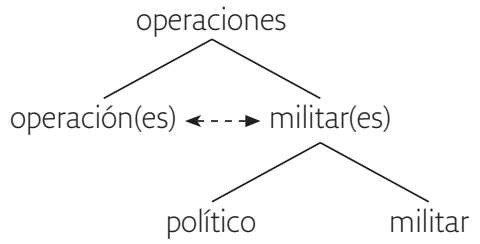

La selección entre una unidad léxica libre (30a) o una unidad ligada (30b) está determinada por las diferencias entre estructuras sintácticas. En (30a) políticas tiene que chequear los rasgos de concordancia de operaciones, pero político en (30b) no lo hace, pues dicho chequeo lo realiza militares. Como en una estructura sintáctica no pueden quedar rasgos sin identificar desde el léxico (Lexicalización Exhaustiva), la forma ligada politico no se podría introducir en una proyección como la de (30a), porque no podría identificar número (*operaciones político rusas militares innecesarias). Tampoco género, pues en compuestos similares como catalano-occidental es evidente que la $o$ que cierra catalano no es un clasificador de género (no existen los *atalanos, sino los catalanes).

En resumen, y partiendo de la propuesta de distinción entre tipos de compuestos en torno a los patrones de acentuación, hemos profundizado en aquellas propiedades que nos permiten distinguir compuestos y sintagmas (expansión de constituyentes, recursividad, etc.). Nuevamente, la imposibilidad de insertar unidades ligadas en entornos funcionales con concordancia ha probado ser de fiabilidad determinante para diferenciar estructuras compositivas y sintagmáticas.

\section{CARACTERIZACIÓN FONOLÓGICA EN TORNO A LA DISTINCIÓN RAÍZ/TEMA}

En el apartado 3.1 veíamos como la presencia de diptongos o la falta de integración fonológica en formas como cuelgacapas conducían a algunos autores a argumentar la existencia de un ciclo de materialización en el interior de compuestos. Igualmente, se postulaba que ciertas características fonológicas de los constituyentes compositivos hacían manifiesta la diferencia entre compuestos con una raíz en posición interna, como berbicida, y compuestos con un tema como bierba-adicto. 
En Fábregas (2004, 303-26) ya encontramos desarrollada esta última idea, o la posibilidad de que las propiedades fonológicas de ciertos constituyentes compositivos en español estén determinadas por su complejidad estructural. En (31) se ejemplifica el tipo de compuestos que presentan como constituyente interno una raíz acategorial; en (32), aquellos que presentan un tema, o conjunto de raíz y categorizador (32):

\section{1. a. coliflor, verdiblanco \\ b. cuellilargo, pasicorto, narilargo}

(Fábregas 2004, 305)

\section{2. castellanohablante; destripaterrones}

(Fábregas 2004, 309)

Se argumenta que solo los constituyentes situados a la izquierda en (31) carecen de autonomía fonológica, alegando que la tendencia del constituyente interno a ser bisilábico y llano es una optimización prosódica que no experimentan los temas de (32). En virtud de dicha optimización se filtran los constituyentes de tres sílabas (33a) y aparecen en su lugar alomorfos como los de (33b). Aunque existen excepciones (33c), estas se consideran palabras marcadas y no productivas.

33. a " naricilargo, ${ }^{*}$ pestañilargo

b. narilargo, cabizbajo

c. cabeciancho, cabeciduro

(Fábregas 2004, 310)

Pese a que existe una tendencia evidente de las formas de (31) a constituir pies binarios, no subscribimos la idea de que este hecho tenga relación alguna con su condición de raíz; de hecho, la propuesta solo se concibe para caracterizar las propiedades prosódicas de un subconjunto de raíces. En el extenso corpus de Buenafuentes (541 hasta final de volumen) encontramos una notable cantidad de formas donde otras raíces -de acuerdo al criterio de ausencia de categorizador- no presentan restricciones silábicas (34a). Respecto a los casos de coordinación, los compuestos de numerales partitivos en (34b) también incumplen dicha restricción en formaciones parasintéticas.

34. a. canceriforme, cavernícola, petrolifero, antenicidio

b. cuarentaitresavo, noventaiochoavo

La situación del tipo de cuellilargo en español contrasta con la del mismo patrón compositivo en catalán (35a), cuyos constituyentes internos tampoco 
constituyen obligatoriamente pies binarios, pues son monosilábicos con frecuencia. Los monosílabos también existen en español, aunque como un producto claro de síncopa vocálica (35b). ${ }^{9} \mathrm{El}$ análisis prosódico justifica, a nuestro entender, la participación de los constituyentes de este compuesto atributivo en una oposición raíz/tema en una lengua como el español, pero no así en una como el catalán. Ello no tendría sentido en un marco teórico neoconstruccionista, donde raíces y temas se diferencian por su complejidad estructural y no por sus particularidades morfofonológicas: las diferencias entre coll-ample y cuelliancho parecen más de tipo morfofonológico que estructural.

35. a. cap-gros, coll-ample

b. manvacío, cabmordido

(Padrosa 248)

La presencia de pies binarios parece ser la pauta silábica por defecto de los sustantivos que aparecen en posición interna en los compuestos del español. Esto se observa al contrastar estos últimos (36) con los verbos y modificadores de (37-38).

36. telediario, compraventa, madreselva marimandona, varapalo, Eurozona

37. destripaterrones, arrebatacapas, despeñaperros

38. donjuan, bienestar, traspatio, sinvergüenza critico-social; alveolo-palatar, americano-argentina

Los ejemplos de (39) están relacionados con castellanobablante, cuyo constituyente interno se consideraba un tema o elemento categorizado.

39. bispanohablante *españolhablante; angloparlante *inglesparlante

Lo que encontramos, a nuestro entender, es que en el esquema de bablante en (39) se optimiza la selección de hispano sobre español, con el resultado de que no se puede afirmar que los compuestos formados con temas no puedan experimentar una optimización prosódica del mismo tipo que los formados con raíces. Para nosotros, una forma como castellano aparecería en castellanobablante por la sencilla razón de que es una forma que no coexiste con alomorfos óptimos. La ausencia de alomorfos óptimos explica también la presencia de cons-

9. La ausencia de $i$ es común en las primeras atestiguaciones del compuesto. En el siglo XIV lo más común era encontrar bocarrasgado, bocabierto, barbapuniente, cuelloalbas, picoabierta. En el siglo XV se imponen ya patitieso, zanquituerto o crestibermejo, según Menéndez Pidal (240). 
tituyentes de tres sílabas en los compuestos sobre "raíces" como espalditendido o caderiancha. Nuestra expectativa es que se pueda producir una optimización prosódica sobre cualquier tipo de unidad léxica ligada (inherente como cuelli o reciclada como castellano), puesto que todos estos compuestos se ajustarían a una misma pauta prosódica, de acuerdo a la propuesta de Elordieta (6-65).

Las unidades ligadas de manera inherente como anglo o mani presentan propiedades morfofonológicas que las diferencian de formas recicladas como cuelga, aunque aparezcan todas ellas en posición interna. Una de dichas propiedades sería la presencia de monoptongos (40a), que, como ya hemos visto, es uno de los argumentos típicos para argumentar su condición de raíces. La aparición de formas monoptongadas es transversal a todos los contextos con unidades ligadas, ya sea en formaciones compuestas o derivadas (40b).

40. a. pernilargo ${ }^{*}$ piernilargo, dentiblancos ${ }^{*}$ dientiblancos

b. pernada, pernera, dentado, desdentar, dentición

La condición común de unidades ligadas de anglo o mani, sin embargo, es insuficiente para explicar importantes diferencias en lo que respecta a sus contextos de inserción. Así, las formas de procedencia clásica presentan distintas variantes alomórficas (41a) y se expanden en la creación contemporánea de tecnicismos en cualquier lengua (41b). La selección de formas patrimoniales como cuelli- o blanqui-, sin embargo, es indiferente a la existencia de alomorfos con antiguas marcas de caso, porque nunca coexisten en el mismo contexto (42a) y no se difunden en otras lenguas como un molde de composición neoclásica (42b).

41. a. manorreductor, maniobrar, manufactura

b. canceriform, patricide, hispanophobia

42. a. *manocorto, manicorto, *manucorto dentifrico, *dentímellado, *dentemellado

b. *bandishort, *dentibroken

Finalmente, aquellos constituyentes identificados por unidades léxicas libres (43) no presentan ningún tipo de optimización prosódica respecto al número de sílabas y admiten la presencia de diptongos, como ejemplifican las formas subrayadas. Sus propiedades serían, por tanto, similares a las de unidades recicladas como destripa, añadiéndose la presencia de acento principal.

43. a. destripaterrones, tragaluz, drogodependencia

b. azul cielo, buevo sorpresa, amenaza fantasma 
En conclusión, la distinción de compuestos como blanquiazul y cuelgacapas en torno a la oposición raíz/tema es problemática por varias razones. En primer lugar, pese a que en la propuesta se aborda un número muy reducido de construcciones, es igualmente posible encontrar excepciones (coliflor, caderiancha). En segundo lugar, la propuesta no puede implementarse sobre casos similares en lenguas relacionadas como el catalán. Finalmente, es contradictoria con uno de los supuestos sobre la oposición raíz/tema de los modelos neoconstruccionistas, para los cuales dicha oposición tendría que tener un correlato con el tipo de significado (idiosincrásico o no) que pudiéramos encontrar en cada uno de los compuestos.

Las formas verbales que aparecen en el compuesto más productivo del español, el que representa matasanos, no solo no presentan competencia con "raíces" ("cidasanos), sino que tampoco compiten con ningún otro tipo de forma verbal (*colgacapas/colgador). Si el contexto de inserción léxica estuviera limitado únicamente a la oposición raíz/tema, *colgacapas sería una forma legítima porque colga evidentemente presenta una vocal temática.

En el análisis en torno a la interfaz entre estructuras y exponentes que presentábamos en el segundo apartado, las particularidades morfofonológicas de los constituyentes internos de compuestos como dentimellado y cuelgacapas constituyen tendencias de materialización, relacionadas tanto con su contexto de inserción como con sus propiedades léxicas intrínsecas. Al representar únicamente tendencias, podemos contemplar excepciones: formas monoptongadas como fregasuelos entre las unidades recicladas, formas con tres sílabas como caderiancha entre las unidades inherentemente ligadas. La posibilidad de explicar las excepciones sin renunciar por ello a la elaboración de generalizaciones hace que el sistema propuesto cuente con mayor adecuación empírica que el de propuestas precedentes.

\section{Conclusiones}

En este trabajo se ha propuesto una nueva tipología de compuestos del español desde la perspectiva de la interfaz entre estructuras sintácticas y exponentes léxicos (unidades libres o ligadas).

La presencia de proyecciones de concordancia se ha relacionado con la aparición de acentos principales, entre otras propiedades fonológicas de las formas. La existencia de este tipo de proyecciones en posición interna ha sido limitada a los compuestos de dos sustantivos de núcleo a la izquierda (camiones cisterna), puesto que el establecimiento de relaciones de concordancia entre elementos léxicos en el primer ensamble se ha destacado como el criterio fundamental para 
distinguir la proyección de compuestos de la de sintagmas (ver Fábregas 2014, 116-17) para una aproximación a la misma cuestión desde el chequeo de rasgos).

Se ha tratado de demostrar que la diversidad de formas existente en los compuestos del español se correlaciona con propiedades de las estructuras, lo que permite unificar y sistematizar el tratamiento de cada categoría gramatical que aparece en un compuesto, una vertiente de análisis que puede ayudarnos a solucionar algunos de los problemas de clasificación de compuestos que permanecen sin resolver de manera satisfactoria.

\section{OBRAS CITADAS}

Abney, Steven P. The English Noun Phrase in its Sentential Aspect. Cambridge: MIT Press, 1987.

Ackema, Peter, y Ad Neeleman. Beyond Morphology: Interface Conditions on Word Formation. Oxford: Oxford UP, 2004.

Baker, Mark C. "The Macroparameter in a Microparametric World". The Limits of Syntactic Variation. Ed. Theresa Biberauer. Ámsterdam: John Benjamins, 2008. 351-73.

Bauer, Laurie. "When is a Sequence of Two Nouns a Compound in English". English Language and Linguistics 2.1 (1998): 65-86.

Bermúdez Otero, Ricardo. "The Spanish Lexicon Stores Stems with Theme Vowels, not Roots with Inflectional Class Features". International fournal of Latin and Romance Linguistics 25.1 (2013): 3-103.

Buenafuentes de la Mata, Cristina. Procesos de gramaticalización y lexicalización en la formación de compuestos en español. Barcelona: UAB, 2007.

Carstairs-McCarthy, Andrew. Current Morphology. Oxford: Routledge, 2002.

De Bustos Gisbert, Eugenio. La composición nominal en español. Salamanca: Universidad de Salamanca, 1986.

Domínguez, Alberto, Fernando Cuetos y Juan Segui. "The Processing of Grammatical Gender and Number in Spanish". Fournal of Psycholinguistic Research 28.5 (1999): 485-98.

Elordieta, Gorka. "The Word in Phonology". To Be or not to Be a Word: New Reflections on the Definition of Word. Eds. Iraide Ibarretxe y José Luis Mendívil Giró. Newcastle: Cambridge Scholars, 2014. 6-65.

Fábregas Alfaro, Antonio. "Prosodic Constraints and the Difference Between Root and Word Compounding”. Lingue e linguaggio 3 (2004): 303-26.

Fábregas Alfaro, Antonio. "On a Grammatically Relevant Definition of Word and Why It Belongs to Syntax". To Be or not to Be a Word: New Reflections 
on the Definition of Word. Eds. Iraide Ibarretxe y José Luis Mendívil Giró. Newcastle: Cambridge Scholars, 2014. 94-130.

Fábregas Alfaro, Antonio. "Stressing about Stress in Compounds". Workshop on the Occasion of Olga Urek's defense (2016a). 18 de noviembre de 2016. $<$ https://castl.uit.no/phocadownload/SpellOut/fabregas.pdf>.

Fábregas Alfaro, Antonio. Las nominalizaciones: la nanosintaxis y la relación entre el léxico y la sintaxis. Madrid: Visor, 2016b.

Halle, Morris, y Alec Marantz. "Distributed Morphology and the Pieces of Inflection". The View from Building 20. Eds. Ken Hale y Samuel Jay Kayser. Cambridge, MA: MIT Press, 1993. 111-76.

Harley, Heidi. "Compounding in Distributed Morphology". The Oxford Handbook of Compounding. Eds. Rochelle Lieber y Pavol Stekauer. Oxford: Oxford UP, 2009. 204-28.

Hualde, José Ignacio. "Stress Removal and Stress Addition in Spanish". Fournal of Portuguese Linguistics 5.1 (2007): 59-89.

Lapesa Melgar, Rafael. "Los casos latinos: restos sintácticos y sustitutos en español”. Boletín de la Real Academia Española 44.310 (1964): 57-106.

Lieber, Rochelle, y Pavol Stekauer, eds. The Oxford handbook of compounding. Oxford: Oxford UP, 2009.

Marchand, Hans. The Categories and Types of Present-Day English Word-Formation: A Synchronic-Diachronic Approach. Múnich: Beck, 1969.

Mendívil Giró, José Luis. "Palabras con estructura externa". Panorama de la lexicología. Ed. Elena de Miguel. Barcelona: Ariel, 2009. 83-116.

Menéndez Pidal, Ramón. Manual de gramática bistórica española. Madrid: Espasa-Calpe, 1941.

Padrosa, Susana. Complex Word-Formation and the Morphology-Syntax Interface. Barcelona: UAB, 2010.

Rainer, Franz, y Soledad Varela Ortega. "Compounding in Spanish". Rivista di linguistica 4.1 (1992): 117-42.

Shwayder, Kobey. "Word-Level Recursion in Spanish Compounds". University of Pennsylvania Working Papers in Linguistics 21.1 (2016). 18 de noviembre de 2016. <http://repository.upenn.edu/cgi/viewcontent.cgi?article $=1857 \&$ context $=$ pwpl $>$.

Starke, Michäl. "Nanosyntax: A Short Primer to a New Approach to Language”. Nordlyd 36.1 (2009): 1-6.

Uriagereka, Juan. "Multiple Spell-Out". Working Minimalism. Eds. Samuel D. Epstein y Norbert Hornstein. Cambridge: MIT Press, 1999. 251-82. 